\title{
A Study of Negative Polarity Items in Chinese Existential Sentences
}

\author{
Wencheng Gao*, Xiaofeng Zhang \\ College of Foreign Languages, University of Shanghai for Science and Technology, Shanghai 200093, China
}

Received September 16, 2020; Revised November 23, 2020; Accepted December 13, 2020

\section{Cite This Paper in the following Citation Styles}

(a): [1] Wencheng Gao, Xiaofeng Zhang, "A Study of Negative Polarity Items in Chinese Existential Sentences," Linguistics and Literature Studies, Vol. 9, No. 1, pp. 12 - 21, 2021. DOI: 10.13189/lls.2021.090102.

(b): Wencheng Gao, Xiaofeng Zhang (2021). A Study of Negative Polarity Items in Chinese Existential Sentences. Linguistics and Literature Studies, 9(1), 12 - 21. DOI: 10.13189/lls.2021.090102.

Copyright $\mathrm{C} 2021$ by authors, all rights reserved. Authors agree that this article remains permanently open access under the terms of the Creative Commons Attribution License 4.0 International License

\begin{abstract}
Negation is crucial to semantics. Negative polarity items (NPI) play an important role in negation. There are a few studies on NPIs in Chinese, but so far no research is on NPIs in Chinese existential sentences (ESs). Because the existential verb "you" (have) is combined with the negative marker "mei" (not) in Chinese ESs, unlike in other types of sentences where "meiyou" (not have) together function as a negative marker. We wonder whether this combination of a single negative marker and an existential verb affects the licensing conditions of NPIs. This is why we study negative polarity items in Chinese ESs. We investigate the variety of negative polarity items which can be allowed in Chinese ESs, and their licensing conditions. It is found that four types of negative polarity items can occur in Chinese ESs, i.e. negative polarity adjectives, negative polarity adverbs, negative polarity wh-words, and negative polarity 'one' phrase as minimizer. In this paper, we just focus on the last two types of negative polarity items that can occur in Chinese ESs. The linguistic facts show that negative polarity wh-words (except 'duoshao') and negative polarity 'one' phrase as minimizer in Chinese ESs can be licensed by negative sentences, yes-no interrogative sentences, A-not-A interrogative sentences, and the antecedent clause of a conditional. We claim that negative polarity wh-words (except 'duoshao') and negative polarity 'one' phrase as minimizer in Chinese ESs are strong NPIs. It has been found that NPIs can be licensed by negative sentences in Chinese according to the previous studies. Our new contribution to the field is that we have found NPIs in Chinese ESs can also be licensed by yes-no interrogatives, A-not-A interrogatives and the antecedent clause of a conditional apart from negative sentences. This finding is to some extent accountable for
\end{abstract}

NPIs in other Chinese constructions.

Keywords Negative Polarity Items, Chinese Existential Sentences, Distribution, Licensing Condition

\section{Introduction}

Negative polarity refers to the grammatical property of a word or phrase, such as ever or any in English that may normally be used only in a semantically or syntactically negative or interrogative context [1]. Words or expressions that either require or shun the presence of a negative element in their context are referred to as negative or positive polarity items (henceforth NPIs and PPIs), respectively. Often examples of NPIs in English are any and yet, while some and already are instances of PPIs. Many languages, perhaps all, have NPIs and PPIs, and their distribution has been the topic of a rapidly growing literature since the seminal work of Klima [2][3]. Negative polarity items also occur in Chinese existential sentences, for example:
(1) Chouti li meiyou renhe dongxi. Drawer in not-have NEG any thing
'There isn't anything in the drawer.'

(2) Qiang shang meiyou renhe dongxi.

Wall on not-have NEG any thing

'There is not anything on the wall.'

(3) Zhe li shenme xiansuo ye meiyou. Here what news either not-have NEG 
'There is not any news here.'

(4) Zhe li yewan meiyou na er ke qu. Here night not-have NEG where may go 'There is no place to go at night here.'

(5) Ta nian shang meiyou yisi xiaorong. He face on not-have NEG one CLF smile 'There is not a trace of smile on his face.'

(6) Zhe ge shichang meiyou yidian er huoli. This CLF market not-have NEG one CLF vitality 'There is not a little vitality in this market.'

Example (1) to (6) are existential sentences, where 'renhe' in example (1), 'renhe' in example (2), 'shenme' in example (3), 'na er' in example (4), 'yisi' in example (5), 'yidian er' in example (6) are negative polarity items, which modify existential entities and occur in negative sentences, enhancing negative effect.

Huang[4] thinks that existential you-sentences in Chinese can be represented as the general form of (NP)...V ...NP...(XP), where NP is optional, either left empty or filled by a locative NP functioning as a subject, $\mathrm{V}$ is filled by the existential verb ' $y o u$ '(have), NP is the existent entity, XP is also optional, which can be filled by either a clause or a phrase predicating over the existent entity. Huang[4] shows that the existential you-sentences in Chinese also exhibit the Definiteness Effect (DE). According to $\mathrm{Hu}$ and $\mathrm{Pan}[5]$, the basic function of Chinese existential you-sentences is to introduce new information into the discourse; the new information can be either a new entity or a new relation, and the so-called Definiteness Effect (DE) is only a by-product of the discourse function of the existential construction. $\mathrm{Gu}[6]$ has proposed a non-movement analysis for the subject of the two types of locative existential construction in Chinese. She suggests that in both types of locative existential construction, the locative subject is base-generated in that position which is theta-marked by the verb. Tsai[7] explores three types of existential quantification in Chinese, you, you-de, you-(yi)-xie, arguing that while presentational you count as a sentential unselective binder, partitive you and specific plural you are to be treated as determiners.

Negation plays an important role in existential quantification. Negative polarity items generally occur in negative environment, which can enhance negative effect. The core research issues about NPIs are the proper delimitation of their distribution and the underlying causes of their distribution. As for the issue of distribution of NPIs, there has been a debate as to whether it should be explored from a syntactic, semantic or pragmatic perspective.

The studies of NPIs run through the history of generative grammar[1]. As for the review of 'negation', the early corpus is mainly focused on English, which has been gradually extended to some other languages in the past 30 years. The empirical evidence across languages indicates that polarity is not only determined by syntactic categories, but also by semantic factors, involving the essence of grammatical structures and language knowledge.

The traditional study on NPI licensing dates back to Klima[1].The issue of NPI licensing is also explored by Progovac[8][9][10], Postal[11], Szabolcsi[12], Dikken[13] and so on, including a syntactic relation between a proper (semi-)negative element and an NPI. Klima[1] proposes a treatment whereby the presence of a morphosyntactic feature [affective] acts as the trigger of a negative polarity item. Among the environments marked as [affective] are: the scope of negation, such as never, nothing, no, none, etc., complements to negative predicates such as unpleasant, unlikely, odd, impossible, etc., comparative clauses, questions, the scope of negative quantifiers and adverbs such as few/little, rarely, barely, hardly, etc.

Progovac[10], for instance, argues that NPI licensing shows significant similarities with syntactic binding. She believes that an NPI is essentially an anaphor that must be A'-bound in its governing category. English 'any' is seen as the subject to Principle A of Binding Theory but is licensed by a superordinate as well as clause-mate negation as it raises in logic form (LF). According to Szabolcsi[12], NPI 'any' and its licenser are the joint spell-out of an underlying negative determiner [D NEG [SOME]]. Dikken[13] believes that at least some NPIs require syntactic agreement with a negative head-in terms of minimalist feature checking.

The studies on negative polarity items tend to circle around the similar examples and items, and focus on various languages, besides English, mainly Dutch, Greek, Italian, Russian, Chinese, Japanese, Korean and Hindi. The scholars try to relate the distribution of NPIs to their meaning.

Ladusaw[14] has proposed to eliminate the feature of [affective] by using a semantic account of the licensing of the polarity items. He points out that many of the contexts in which polarity items are acceptable have the property of downward monotonicity or implication reversal. Normally, expressions may be replaced by more general ones salva veritate, for example, 'John is a sophomore.' will entail 'John is a university student.', given that a sophomore is the second-year university student. Now for the negative counterpart of these sentences, the direction of the entailment is reversed: 'John is not a university student.' entails 'John is not a sophomore.' but not vice versa in propositional logic, which can be represented as $\mathrm{p} \rightarrow \mathrm{q},-\mathrm{q} \rightarrow-\mathrm{p}$. Here, the proposition $\mathrm{p}$ is 'John is a sophomore', the proposition $\mathrm{q}$ is 'John is a university student.', $\mathrm{p}$ entails q. And the negation of $\mathrm{q}$ entails the negation of $\mathrm{p}$, because 'sophomore' is a lower word of the upper word 'university student'. However, the reversed negation is not necessarily correct in propositional logic, for instance, 'John is not a sophomore.' does not 
necessarily entail 'John is not a university student', maybe he is a freshman.

Zwarts[15], Nam[16], and van der Wouden[17] have argued that the typology of NPIs corresponds to a typology of 'monotone decreasing' operators, which can be summarized: a. Weak NPIs are licensed by decreasing functions, like 'any' 'ever' in English; b. Strong NPIs are licensed by anti-additive functions, like ' $y e t$ ' in English; c. Strongest NPIs are licensed by anti-morphic functions, like ' $a$ bit' in English. According to the above typology, different downward-entailing operators license different NPIs. There is a subset relation among the three types of NPIs. For example, anti-additive operators are a proper subset of monotone decreasing operators, and anti-morphic operators are a proper subset of the anti-additive operators.

However, negative polarity items in Chinese ESs are overlooked. Many issues concerning NPIs in Chinese ESs are worth exploring, such as the variety, the distribution, and the licensing conditions of NPIs in Chinese ESs, and how they interact on the three levels of syntax, semantics and pragmatics. Since the first two types of negative polarity items that can occur in Chinese ESs, negative polarity adjectives and negative polarity adverbs, have been investigated in the first paper, we will focus on the third and fourth types of negative polarity items in this paper, i.e. negative polarity wh-words and negative polarity "one" phrase as minimizer.

Huang's definition to Chinese existential sentence is particularly defined from a perspective of formal linguistics. We provide our definition before we address the two types of NPIs in Chinese ESs. Existential sentence is the sentence pattern which expresses the existence of something or somebody in some place, whose pattern can be summarized as "some place has something or someone". Another similar sentence pattern is (dis)appearing sentence (yinxian $j u$ ). (Dis) appearing sentence is the sentence pattern which expresses someone or something appears or disappears in some place, whose pattern can be summarized as "some place appears or disappears someone or something". In its broad sense, existential sentences refer to existential sentences and (dis)appearing sentences; in its narrow sense, (dis)appearing sentences are excluded. In this paper we use existential sentences in its narrow sense.

We wonder whether the combination of a single negative marker ' $m e i$ ' (not) and an existential verb 'you' (have) affects the licensing conditions of NPIs in Chines ESs. In other types of sentences, 'meiyou' (not) together functions as a compound negative syntactic marker. There are three research questions in this paper: 1) Does the combination of a single negative marker and the existential verb affect the licensing conditions of NPIs in Chinese ESs? 2) What types of NPI can be allowed in Chinese ESs? 3) What are their licensing conditions respectively?

This paper consists of four sections. In section one, we briefly introduce the literature on the studies of Chinese ESs and the research of NPI abroad. In section two, we will discuss negative polarity wh-words in Chinese ESs in detail. In section four, negative polarity "one" phrase as minimizer in Chinese ESs will be explored in detail. The last section is conclusion.

\section{Negative Polarity Wh-words in Chinese Existential Sentences}

Wh-words in Chinese have interrogative function, and they can also be used as negative polarity items. Chinese wh-words such as 'shenme'(what), 'shei'(who), 'na'er' (where), etc., may sometimes be interpreted as non-interrogative existential indefinites, meaning 'something', 'somebody', 'somewhere', etc. These existential wh-words typically occur in negative sentences such as 'wo mei chi shenme' (I didn't eat anything), 'wo mei zhao shei' (I didn't look for anybody), 'wo mei qu na'er' (I didn't go anywhere), etc. However, they cannot occur in affirmative sentences in these senses, such as * 'wo chi le shenme' (I ate something), *'wo zhao le shei' (I looked for somebody), * 'wo qu le na'er' (I went somewhere), etc. Some scholars treat non-interrogative existential wh-words as polarity items [18][19][20][21]. The purpose of this section is to investigate the distribution and licensing of existential wh-words in Chinese ESs where such existential wh-words are used as negative polarity items.

Chinese speakers tend to use wh-words, such as 'shenme' (what), 'shui' (who), 'na'er' (where), etc., to express emphasis on negation. Chinese wh-words may show a thorough negation and function as NPIs in Chinese ESs, for example: 
(7) Bingxiang li meiyou shenme dongxi.

Refrigerator in not-have NEG what thing

'There is not anything in the refrigerator.'

In example (7), 'shenme' is not used as an interrogative wh-word but as NPI, which is licensed by the negative marker 'mei(you)'. In this context, NPI 'shenme' has insignificance reading, used to modify the existential entity 'dongxi'.

NPI 'shenme' can also be understood in the sense of none reading, for example:

(8) Bingxinag li shenme dongxi ye/dou meiyou.

Refrigerator in what thing either/all not-have NEG

'There is nothing in the refrigerator.'

In example (8), NPI 'shenme' is used together with the focus adverb ' $y e$ ' or 'dou', which makes the sentence order changed with the object of existential verb going from the right to the left. In this context, NPI 'shenme' is interpreted as none reading, meaning 'nothing'.

NPI 'shei' and ' $n a$ er' may also occur in Chinese ESs, for instance:

(9) Zhe er meiyou shei yuanyi qu.

Here not-have NEG who willing go

'Nobody is willing to go here.'

(10) Zheli yewan meiyou na er ke qu.

Here night not-have NEG where may go

'There is no place to go at night here.'

In example (9), NPI 'shei' is licensed by the negative marker 'mei(you)', and it is understood in the sense of 'nobody'. In example (10), NPI ' $n a$ er' is also licensed by the negative marker 'mei(you)', and it is understood in the sense of 'no place'. Unlike example (4), when the object of the existential verb in example (10) is focalized, NPI 'shei' requires the collocation of the focus adverb ' $y e$ ' or 'dou', meanwhile the negative marker 'mei(you)' has to be changed into 'bu', as shown in example (11). The same is true of NPI ' $n a$ er' in example (10), which can be illustrated by example (12).

(11) Zhe er shei ye/dou bu yuanyi qu. Here who either/all not NEG willing go

'Nobody is willing to go here.'

(12) Zheli yewan na er ye/dou qu bu le. Here night where either/all go not NEG ASP

'There is no place to go at night here.'

NPI 'shenme', 'shei', and ' $n a$ er' also occur in yes-no interrogative sentences, for example:

(13) Zhe er you shenme chi de ma?

Here have what eat DE Q?

'Is there something to eat here?'

(14) Zhe er you shei yuanyi canjia ma?

Here have who willing join Q?

'Is there somebody who is willing to join us here?'

(15) Zhe er you na er keyi xiu biao ma?

Here have where can repair watch Q?

'Is there some place where I can repair my watch here?'

NPI 'shenme' in example (13) is licensed by the interrogative sentence, and it is used as an object of the existential verb 'you' (have) with the meaning of 'something'.

Based on the method used by Higginbotham[22], Cai[23] shows that yes-no question sentence in Chinese can be a licensing context of NPI 'renhe', and yes-no question sentence and A-not-A question sentence both have semantic characterization of anti-additivity. Anti-additive environments are a proper subset of downward entailment contexts, and anti-morphic environments are a proper subset of anti-additive contexts. Negative sentences and interrogatives can license similar items because they have downward entailment contexts, which are not shared by affirmative sentences.

NPI 'shei' in example (14) is also licensed by the interrogative sentence, and it is used as an object of the existential verb 'you' with the meaning of 'somebody'. NPI 'na er' in example (15) is also licensed by the interrogative sentence, 
and it is used as an object of the existential verb ' $y o u$ ' with the meaning of 'some place'.

Negative polarity wh-words 'shenme', 'shei', and 'na er' also appear in A-not-A interrogative sentences, for instance:

(16) Zheli you-mei-you shenme zhongyao xiansuo?

Here have-not-have what important clue

'Are there any important clues here or not?'

(17) Zheli you-mei-you shei zhidao zhenxiang?

Here have-not-have who know truth

'Is there somebody who knows the truth here or not?'

(18) Zheli you-mei-you na er keyi chu qu?

Here have-not-have where can go out

'Is there some place where we can go out here or not?'

NPI 'shenme' in example (16) is licensed by A-not-A interrogative sentence, and it is used as a modifier of the existential noun 'xiansuo', meaning 'any'. NPI 'shei' in example (17) is also licensed by A-not-A interrogative sentence, but it is used as an object of the existential verb 'you', meaning 'somebody'. In example (18), NPI ' $n a$ er' is also licensed by A-not-A interrogative sentence, and it is used as an object of the existential verb 'you', meaning 'some place'.

NPI 'shenme', 'shei', and 'na er' also occur in the antecedent clause of a conditional, for example:

(19) Ruguo zhe er you shenme chi de, qing gei women yidian er.

If here have what eat DE please give us a little

'If there is something to eat here, please give us a little of them.'

(20) Ruguo zhe er you shei yuanyi canjia, qing gaoshu wo.

If here have who willing join please tell me

'If there is somebody who is willing to join us here, please tell me.'

(21) Ruguo zhe er you na er keyi xiu biao, qing gaoshu wo.

If here have where can repair watch please tell me

'If there is some place where I can repair my watch, please tell me.'

Example (19) is a conditional, and NPI 'shenme' appears in the antecedent clause of it. NPI 'shenme' is used as an object of the existential verb ' $y o u$ ', and it is licensed by the antecedent clause of a conditional, meaning 'something'. In example (20), NPI 'shei' is used as an object of the existential verb 'you', which is also licensed by the antecedent clause of a conditional, meaning 'somebody'. Similarly, in instance (21), NPI ' $\mathrm{na}$ er' is also licensed by the antecedent clause of a conditional, meaning 'some place'.

Apart from these three negative polarity wh-words, 'shenme', 'shei', and 'na er', there is another wh-word 'duoshao' (how many), which can also be used for non-interrogative function. For instance, 'wo meiyou duoshao jihui' (I don't have much chance), in this sentence 'duoshao' means 'much' in a negative sentence. It cannot appear in an affirmative sentence in this sense, for example, *'wo you duoshao jihui' (I have much chance). NPI 'duoshao' occurs in negative existential sentences, expressing indefinite quantity, for instance:

(22) Zheli meiyou duoshao you jiazhi de ziliao.

Here not-have NEG how many have value DE materials

'There are not many valuable materials here.'

In example (22), negative polarity wh-word 'duoshao' is licensed by the negative marker 'mei(you)'. NPI 'duoshao' is used as a modifier of the existential noun 'ziliao' (materials), in this context it expresses indefinite quantity, in the sense of insignificance reading, meaning 'not many'. However, NPI 'duoshao' cannot occur in yes-no question sentences, A-not-A interrogative sentences, and even the antecedent clauses of a conditional, for example:

(23) * Zheli you duoshao you jiazhi de ziliao ma?

Here have how many have value DE material Q

*'Are there how many valuable materials here?'

(24) *Zheli you-mei-you duoshao you jiazhi de ziliao?

Here have-not-have how many have value DE material

*'Are there how many valuable materials here or not?' 
(25)* Ruguo zheli you duoshao you jiazhi de ziliao, wo jiu ban $k a$.

If here have how many have value DE material, I then apply for card

*'If there are how many valuable materials here, then I'll apply for a card.'

Example (23) is not acceptable, as we can see from this instance, NPI 'duoshao' is not licensed by the interrogative sentence. Example (24) is not acceptable, either, where NPI 'duoshao' is not licensed by the A-not-A interrogative sentence. In example (25), NPI 'duoshao' appears in the antecedent clause of a conditional, but the whole sentence is not accepted. The unacceptability of the sentence means NPI 'duoshao' is not licensed by the antecedent clause of a conditional.

To summarize, negative polarity wh-words in Chinese ESs, such as 'shenme', 'shei', 'na'er', etc. are licensed by the negative marker 'mei(you)'. They can also be licensed by yes-no question sentences, A-not-A interrogative sentences, and the antecedent clauses of a conditional. In such contexts they are used as NPIs. However, negative polarity wh-word 'duoshao' can only be licensed in negative existential sentences, while in other contexts such as yes-no question sentences, A-not-A interrogative sentences, and the antecedent clauses of a conditional, NPI 'duoshao' is not licensed.

\section{Negative Polarity 'One' Phrase as Minimizer in Chinese Existential Sentences}

In addition, there exists another kind of negative polarity determiner structure 'one' phrase as minimizer in Chinese ESs. There are a number of unit words in Chinese, which can collocate with the basic numeral ' $y i$ ' (one), expressing minimal quantity. So we call this kind of structure 'one' phrase as minimizer, such as 'yidian'er', 'yige', 'yitai', 'yisi', 'yihao', 'yijin', 'yiliang', 'yichi', 'yicun', 'yizhi', 'yigen', 'yibu', etc. They are all expressions composed of 'yi' and various classifiers in Chinese, which are used with a noun together to minimize the quantity of the existential entity in ESs.

Let us look at the distribution and licensing of the frequently used 'one' phrase as minimizer 'yidian er' (a little) in Chinese ESs, for example:

(26) Xiangmu meiyou yidian er jinzhan.

Project not-have NEG a little progress

'There is not a little progress in the project.'

(27) Xiangmu you yidian er jinzhan ma?

Project have a little progress Q

'Is there a little progress in the project?'

(28) Xiangmu you-mei-you yidian er jinzhan?

Project have-not-have a little progress

'Is there a little progress in the project or not?'

(29) Ruguo xiangmu you yidian er jinzhan, women jiu geng you xinxin le.

If project have a little progress we then more have confidence LE

'If there is a little progress in the project, then we have more confidence.'

In example (26), NPI 'yidian er' (a little) is used as a modifier of the existential noun 'jinzhan' (progress), and it is licensed by the negative marker 'mei(you)'. In example (27), NPI 'yidian er' is also used as a modifier of the existential noun, but it is licensed by a yes-no interrogative sentence rather than a negative marker. In example (28), NPI 'yidian er' is also used as a modifier of the existential noun, but it is licensed by A-not-A interrogative sentence. In example (29), used as a modifier of the existential noun, NPI 'yidian er' is licensed by the antecedent clause of a conditional.

The second frequently used 'one' phrase as minimizer in Chinese ESs is 'yige' (one + classifier), for instance:

(30) Yiyuan li meiyou yige hugong.

Hospital interior not-have NEG one CLF nursing worker

'There is not a nursing worker in the hospital.'

(31) Yiyuan li you yige hugong ma? Hospital interior have one CLF nursing worker Q

'Is there a nursing worker in the hospital?'

(32) Yiyuan li you-mei-you yige hugong?

Hospital interior have-not-have one CLF nursing worker 
'Is there a nursing worker in the hospital or not?'

(33) Ruguo yiyuan li you yige hugong, wo jiu qing ta bangmangle

If hospital interior have one CLF nursing worker I then ask him help ASP

'If there was a nursing worker in the hospital, I would ask him to give me a hand.'

In example (30), NPI 'yige' (one + classifier) is used as a modifier of the existential noun 'hugong' (nursing worker), and it is licensed by the negative marker 'mei(you)'. In example (31), NPI 'yige' remains as a modifier of the existential noun, but it is licensed by the yes-no interrogative sentence, which clearly expresses negative meaning. In example (32), NPI 'yige' is also used a modifier of the existential noun, but it is licensed by A-not-A interrogative sentence. Though example (32) is A-not-A interrogative sentence, it is in general employed to express negative meaning. In example (33), NPI 'yige' is still used as a modifier of the existential noun, but it is licensed by the antecedent clause of a conditional, which also conveys negative implicature.

The third frequently used 'one' phrase as minimizer in Chinese ESs is 'yisi' (one + classifier), for instance:

(34) Tade lian shang meiyou yisi xiaorong.

His face on not-have NEG one CLF smile

'There is not a trace of smile on his face.'

(35) Tade lian shang you yisi xiaorong ma?

His face on have one CLF smile Q

'Is there a trace of smile on his face?'

(36) Tade lian shang you-mei-you yisi xiaorong?

His face on have-not-have one CLF smile

'Is there a trace of smile on his face or not?'

(37) Ruguo tade lian shang you yisi xiaorong, na ye suan ta you limao. If his face on have one CLF smile that either count he have polite 'If there is a trace of smile on his face, that means he is polite.'

In example (34), NPI 'yisi' (one + classifier) is used as a modifier of the existential noun 'xiaorong' (smile), which means 'a trace of', and it is licensed by the negative marker 'mei(you)'. In example (35), NPI ' $y i s i$ ' is also employed as a modifier of the existential noun, but it is licensed by the yes-no interrogative sentence. Though this sentence is made in the form of a question, but it expresses negative meaning. In example (36), used a modifier of the existential noun, NPI 'yisi' is licensed by A-not-A interrogative sentence, which generally expresses negative meaning. In example (37), NPI 'yisi' is still used as a modifier of the existential noun, but it is licensed by the antecedent clause of a conditional. In general, this conditional is employed to convey negative implicature. The tree diagram of example (34) can be shown as the following:

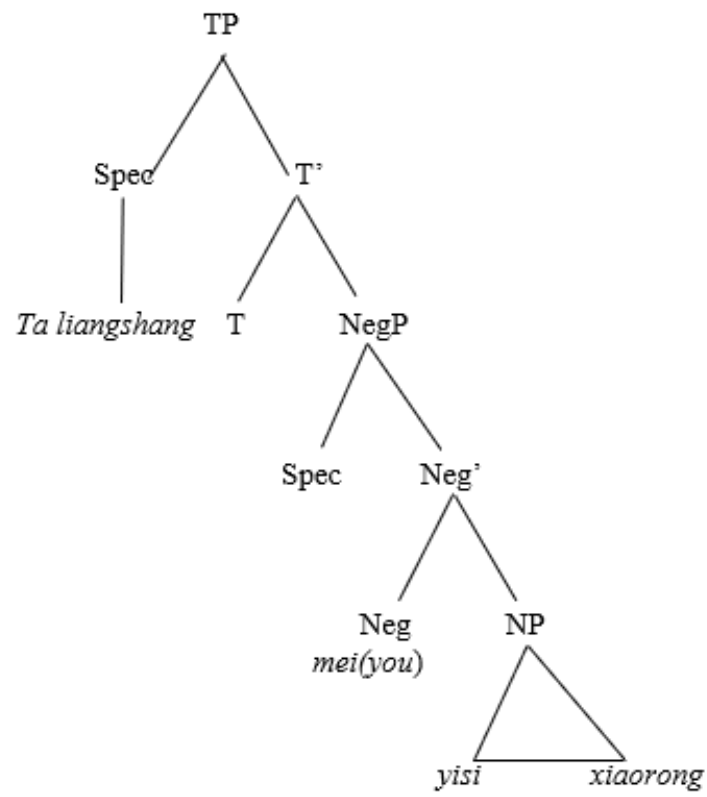


The fourth 'one' phrase as minimizer in Chinese ESs that I will investigate is 'yitai' (one + classifier), for instance:

(38) Gang jian chang shi, chejian li meiyou yitai jiqi. First build factory when, workshop in not-have NEG one CLF machine 'When the factory was first built, there was not a machine in the workshop.'

(39) Gang jian chang shi, chejian li you yitai jiqi ma? First build factory when, workshop in have one CLF machine Q 'When the factory was first built, was there a machine in the workshop?'

(40) Gang jian chang shi, chejian li you-mei-you yitai jiqi? First build factory when, workshop in have-not-have one CLF machine 'When the factory was first built, was there a machine in the workshop or not?'

(41) Ruguo gang jian chang shi, chejian li you yitai jiqi, na suan haode. If first build factory when, workshop in have one CLF machine, that count good 'When the factory was first built, if there was a machine in the workshop, that would be good.'

In example (38), NPI 'yitai' (one + classifier) is used as a modifier of the existential noun ' $j i q i$ ' (machine), and it is licensed by the negative marker 'mei(you)'. In example (39), NPI 'yitai' is also employed as a modifier of the existential noun, but it is licensed by the yes-no interrogative sentence. Though this sentence is made in the form of a question, but it actually expresses negative meaning. In example (40), used a modifier of the existential noun, NPI 'yitai' is licensed by A-not-A interrogative sentence, which also generally expresses negative meaning. In example (41), NPI 'yitai' is still used as a modifier of the existential noun, but it is licensed by the antecedent clause of a conditional. This conditional is generally employed to convey negative meaning.

By using the focus adverb 'ye' or 'dou', the existential nouns and its modifiers acted by NPIs such as 'yidian'er', 'yige', 'yisi', 'yitai', can be focalized by moving from a postverbal position to a preverbal position, thus further reinforcing negative effect of the existential sentence, which can be illustrated as follows:

(42) Xiangmu yidian'er jinzhan ye/dou meiyou .

Project a little progress either/ all not-have NEG

'There is not a little progress in the project at all.'

(43) Yiyuan li yige hugong ye/dou meiyou. Hospital in one CLF nursing worker either/all not-have NEG

'There is not a nursing worker in the hospital at all.'

(44) Tade lian shang yisi xiaorong ye/dou meiyou.

His face on one CLF smile either/all not-have NEG

'There is not a trace of smile on his face at all.'

(45) Gang jian chang shi, chejian li yitai jiqi ye/dou meiyou .

First build factory when, workshop in one CLF machine either/all not-have NEG

'When the factory was first built, there was not a machine in the workshop at all.' 
To sum up, NPI 'yidian er', 'yige', 'yisi', and 'yitai' in Chinese ESs often occur as a modifier of the existential noun in negative sentences, expressing minimal quantity or total negation. " $Y i$ " (one) is the basic number in Chinese, and functions as minimal quantity limiter which restrains the quantitative scope of the whole expression. " $Y i$ " remains the prerequisite of "One" phrase minimizer. It must collocate with minimal quantity classifier, such as "si" (silk), "hao" (millimeter), "dian" (point), "ge" (classifier of concrete object), "tai" (classifier of machine), etc. In this case it can never collocate with maximal quantity classifier, such as "dei" (pile), "che" (truck), "changku" (warehouse), etc. The following noun may be an abstract noun or a concrete noun. Whatever the following noun is, the basic number "yi" should be the prerequisite and the classifier ought be minimal quantity one. The semantic congruity and grammatical collocation require the combination of " $y i$ " and minimal quantity classifiers, which gives rise to "one" phrase minimizer, expressing very strong negative effect.

By using the focus adverb ' $y e$ ' or 'dou', the existential noun and its modifier acted by NPIs can be focalized by changing the sentence order, from the right to the left to the negative marker 'mei(you)', thus strengthening negation. Apart from negative sentences, the four NPIs can also be licensed by yes-no interrogative sentences, A-not-A interrogative sentences and the antecedent clauses of a conditional.

\section{Conclusions}

Two types of NPIs in Chinese ESs, namely, negative polarity wh-words and negative polarity 'one' phrase as minimizer are investigated. Including the first two types of NPIs which can be allowed in Chinese ESs, i.e. negative polarity adjectives and negative polarity adverbs, which have been studied in the first paper, there are four types of NPIs that can occur in Chinese ESs. We just summarize the third and fourth types of NPIs in Chinese ESs here, in particular, their licensing conditions.

The combination of a single negative marker and the existential verb does not affect the licensing conditions of NPIs in Chinese ESs. The single negative marker 'mei' functions in the same way as the compound negative marker 'meiyou' in other types of sentences.

The third type of NPI which can be allowed in Chinese ESs is non-interrogative wh-words, such as 'shenme', 'shei', 'na'er', 'duoshao', etc. Negative polarity wh-words in Chinese ESs are mainly employed as objects or modifiers of an object after the existential verb. Negative polarity wh-words in Chinese ESs are licensed by negative sentences, yes-no interrogative sentences, A-not-A interrogative sentences, and the antecedent clauses of a conditional. Among them, NPI of wh-word 'duoshao' is only licensed by negative sentences, but it is not licensed by yes-no interrogative sentences, A-not-A interrogative sentences, and the antecedent clauses of a conditional. Therefore, NPI 'shenme', 'shei' and 'na'er' in Chinese ESs are strong negative polarity items, while NPI 'duoshao' is a weak one. In some cases, NPIs of wh-words and the existential nouns can be focalized by using the focus adverb ' $y e$ ' or 'dou', by which the position of the existential noun phrase is converted from after the existential verb to before it, increasing the salience of the existential noun phrase.

The fourth type of NPI which can occur in Chinese ESs is 'one' phrase as minimizer, such as 'yidian'er', 'yige', 'yisi', 'yitai', etc. Negative polarity 'one' phrases as minimizers are mainly employed as modifiers of the existential nouns, expressing minimal quantity or total negation. NPIs of 'one' phrase as minimizer in Chinese ESs are licensed by negative sentences, yes-no interrogative sentences, A-not-A interrogative sentences, and the antecedent clauses of a conditional. Such NPIs and the existential nouns can also be focalized by using the focus adverb ' $y e$ ' or 'dou', by which the position of the existential noun phrase is converted from the postverbal position to the preverbal one, becoming more salient and strengthening the negation. The linguistic fact that NPIs, sometimes with the existential noun, in Chinese ESs can be focalized, provides us with more examples of language interface interaction.

\section{Funding}

This study is funded by the Planning Fund of Humanities and Social Sciences from Ministry of Education of China (NO. 17YJA 740012), 'From a Cognitive Perspective: A Contrastive Study of Existential Sentences in English and Chinese Based on Corpus'.

\section{REFERENCES}

[1] Dictionaries, C., Forsyth, M. \& Mangan, L. 2014. Collins English Dictionary: Complete and Unabridged, $12^{\text {th }}$ edition. Harpers Collins Publishers.

[2] Klima, E.1964. Negation in English. In J. Fordor and J. Katz (eds.) The Structure of Language. Englewood Cliffs, NJ: Prentice-Hall, 246-323.

[3] Hoeksema, J. 2006. Polarity Items. Encyclopedia of Language and Linguistics, 658-661.

[4] Huang, C.-T.-J.1987. Existential sentences in Chinese and (in)definiteness. In E. Reuland \& A. ter Meulen (eds.) The Representation of (In)definiteness, 226-253. Cambridge \& London: The MIT Press.

[5] Hu Jianhua. \& Pan Haihua. 2008. Focus and the Basic Function of Chinese Existential You-Sentences. In I. Comorovski and $\mathrm{K}$. von Heusinger (eds.). Existence: Semantics and Syntax, 133-145. 
[6] Gu, Y.1992. On the Locative Existential Construction in Chinese. In Dawn Bates (eds.). The Tenth West Coast Conference on Formal Linguistics, 183-195. Stanford: The Stanford Linguistics Association.

[7] Tsai, W.T. D. \& Chang, M.Y. 2003. Two Types of Wh-adverbials: A Typological Study of. Linguistic Variation Yearbook, 3(1): 213-236.

[8] Progovac, L. 1992. Negative Polarity: A Semantic-Syntacti c Approach. Lingua, 86(4), 271-299.

[9] Progovac, L.1993. Negative Polarity: Entailment and Binding. Linguistics and Philosophy, 16 (2),149-180.

[10] Progovac, L.1994. Negative and Positive Polarity: A Binding Approach. Cambridge: Cambridge University Press.

[11] Postal, P. 2000. The SQUAT Lectures, M.S. New York University.

[12] Szabolsci, A. 2004. Positive Polarity-Negative Polarity. Natural Language \& Linguistic Theory, 22(2), 409-452.

[13] Dikken, D. 2002. Direct and Parasitic Polarity Item Licensing. The Journal of Comparative Germanic Linguistics, 5, 35-66.

[14] Ladusaw, W.1979. On the Notion Affective in the Analysis of Negative Polarity Items. Paper presented to the 1979
Annual Meeting of the LSA, Los Angeles.

[15] Zwarts, F. 1990. The Syntax and Semantics of Negative Polarity. Views on the Syntax-Semantics Interface II, Berlin.

[16] Nam, S.1994. Another Type of Negative Polarity Item. In Makoto Kanazawa and Christopher Pinon (eds.). Dynamics, Polarity, and Quantification, CSLI, Stanford, 3-17.

[17] van der Wouden, T.1994. Negative Contexts. Groningen: Groningen Dissertations in Linguistics 12.

[18] Huang, C.T. J. 1982. Move WH in a Language without wh-movement. The Linguistic Review 1, 369-416.

[19] Cheng, Lisa. 1991. On the Typology of Wh-questions. Ph.D. Dissertation, MIT.

[20] Cheng, Lisa. 1994. Wh-words as Polarity Items. Languages in China and Linguistics, 2, 616-640.

[21] Li Yenhui, Audrey. 1992. Indefinite Wh- in Mandarin Chinese. Journal of East Asian Linguistics, (1), 125-155.

[22] Higginbotham, J. 1996. The Semantics of Questions. In S. Lappin (ed.). The Handbook of Contemporary Semantic Theory. Oxford: Blackwell, 361-383.

[23] Cai, Wenzhu. 2010. Semantic Processing of Licensing Conditions of Negative Polarity Items. The Journal of the Second Beijing College of Foreign Languages, (12): 24-28. 